\title{
Primary primitive neuroectodermal tumor of the cervix
}

This article was published in the following Dove Press journal:

OncoTargets and Therapy

15 June 2013

Number of times this article has been viewed

Bo $\mathrm{Li}^{\prime}$

Ling Ouyang'

Xue Han'

Yang Zhou'

Xin Tong'

Shulang Zhang'

Qingfu Zhang ${ }^{2}$

'Department of Obstetrics and Gynecology, Shengjing Hospital of China Medical University, ${ }^{2}$ Department of Pathology, the First Affiliated Hospital and College of Basic Medical Sciences, China Medical University, Shenyang,

People's Republic of China
Correspondence: Ling Ouyang

Department of Obstetrics and Gynecology, Shengjing Hospital of China Medical University, Shenyang II 0004, People's Republic of China Email ouyang1964@163.com
Abstract: Primary primitive neuroectodermal tumors (PNETs) are rare and high-grade malignant tumors that mostly occur in children and young adults. The most common sites are the trunk, limbs, and retroperitoneum. Herein, we present a case of a PNET involving the cervix uteri in a 27-year-old woman. The lesion showed characteristic histologic features of a PNET and was positive for the immunohistochemical markers cluster of differentiation (CD) 99 , vimentin, neuron-specific enolase, neural cell adhesion molecule 1 (CD56), and CD117 (c-kit), further defining the tumor while helping to confirm PNET. The clinical Stage IIIB tumor was treated with chemotherapy and radiotherapy.

Keywords: primitive neuroectodermal tumor, PNET, cervical neoplasm, immunohistochemistry

\section{Introduction}

Primitive neuroectodermal tumors (PNETs) are a type of small round cell tumor that originate from the primitive neuroectoderm and most commonly arise in the chest wall and paraspinal regions. Although PNETs can occur in a number of organs, such as in the kidneys, ${ }^{1}$ brain, ${ }^{2}$ and lungs, ${ }^{3}$ they also occur very rarely in the cervix. As far as we are aware, only nine cases have been reported. PNETs are high-grade malignant, invasive tumors. Currently, there is no uniform standard treatment protocol. Herein, we present a case of primary cervical PNET and describe the diagnosis and treatment process to increase awareness of this condition.

\section{Materials and methods}

We used $4 \mu \mathrm{m}$ sections of formalin-fixed, paraffin-embedded tissue for routine light microscopy examination and immunohistochemical (IHC) analysis. Antibodies against cluster of differentiation (CD) 99, pan-cytokeratin, vimentin, neuron-specific enolase (NSE), neural cell adhesion molecule 1 (CD56), CD117, thyroid transcription factor 1 (TTF-1), terminal deoxynucleotidyl transferase (TdT), leukocyte common antigen (CD45), common acute lymphoblastic leukemia antigen CD10, CD38, myogenic markers (desmin and myogenin), markers of malignant melanoma (S-100 and HMB45), and Ki-67 (ready to use, Fuzhou Maixin Biotechnology Development, Fuzhou, People's Republic of China) were used for IHC analysis (Table 1). The sections were incubated with the primary antibody at $4^{\circ} \mathrm{C}$ overnight before being treated with $3 \%$ $\mathrm{H}_{2} \mathrm{O}_{2}$ and $5 \%$ rabbit serum at $37^{\circ} \mathrm{C}$ for 1 hour. Next, the sections were incubated with the secondary antibody and streptavidin-peroxidase (SP) complex for 30 minutes using an SP kit (Fuzhou Maixin Biotechnology Development). Slides were visualized using 
Table I Antibodies used

\begin{tabular}{|c|c|c|c|c|c|}
\hline Antibody & Clone & Product no & Clonality & Positive location & Antigen retrieval \\
\hline CD99 & 013 & MAB-0059 & Monoclonal & Cell membrane/cytoplasm & Citrate buffer heat repairing \\
\hline Pan-cytokeratin & MX005 & MAB-067I & Monoclonal & Cytoplasm & Citrate buffer heat repairing \\
\hline Vimentin & SP20 & RMA-0547 & Polyclonal & Cytoplasm & Citrate buffer heat repairing \\
\hline NSE & E27 & MAB-0584 & Monoclonal & Cytoplasm & Citrate buffer heat repairing \\
\hline CD56 & $56 C 04$ & MAB-0256 & Monoclonal & Cell membrane & Citrate buffer heat repairing \\
\hline CDII7 & YRI45 & RMA-0632 & Polyclonal & Cell membrane/cytoplasm & EDTA heat repairing \\
\hline TTF-I & MX0II & MAB-0677 & Monoclonal & Cell nucleus & Citrate buffer heat repairing \\
\hline TdT & MX010 & MAB-0676 & Monoclonal & Cell nucleus & EDTA heat repairing \\
\hline CD45 & $\mathrm{PD} 7 / 26+2 \mathrm{BII}$ & MAB-0037 & Monoclonal & Cell membrane & Citrate buffer heat repairing \\
\hline CD38 & $38 \mathrm{CO} 3$ & MAB-034I & Monoclonal & Cell membrane & Citrate buffer heat repairing \\
\hline Desmin & D33 & MAB-0055 & Monoclonal & Cytoplasm & Citrate buffer heat repairing \\
\hline Myogenin & F5D & MAB-0362 & Monoclonal & Cell nucleus & Citrate buffer heat repairing \\
\hline S- 100 & $4 c 4.9$ & Kit-0007-2 & Monoclonal & Cytoplasm & Citrate buffer heat repairing \\
\hline HMB45 & MAB-0359 & HMB45+50 & Monoclonal & Cytoplasm & Citrate buffer heat repairing \\
\hline Ki-67 & MAB-0672 & MAB-0672 & Monoclonal & Cell nucleus & Citrate buffer heat repairing \\
\hline
\end{tabular}

Abbreviations: CD, cluster of differentiation; EDTA, ethylenediaminetetraacetic acid; NSE, neuron-specific enolase; TTF-I, thyroid transcription factor I; TdT, terminal deoxynucleotidyl transferase; HMB-45, human black melanoma 45; S-100, S100 calcium binding protein.

3,3-diaminobenzidine. Sections were stained with nonspecific antibodies to provide a negative control for each reaction.

\section{Case description}

A 27-year-old woman presented with contact bleeding, which she had experienced more than 3 months. At first, the bleeding was slight, guttate, scarlet, and would stop on its own, so the patient paid no attention to it. Further, 10 days before presenting to the hospital, she experienced yellow vaginal discharge with no special odor. She also suffered from occasional lower abdominal pain, without waist pain. Therefore, she visited the Department of Obstetrics and Gynecology, Shengjing Hospital of China Medical University.

Pelvic examination revealed that the vulva had developed normally, but there was evidence of vaginal stenosis. Many nodular lesions were observed on the vagina anterior wall. These extended to the lower third of the vagina and were dark grey and hard. The cervix had lost its normal form and could not be exposed completely. The surface of the cervix was not smooth and had necrotic tissue, which showed evidence of contact bleeding. Tri-manual examination revealed bilateral cardinal ligament thickening and shortening with poor elasticity, which reached the pelvic wall. The values for serum tumor markers such as carcinoembryonic antigen $(0.4 \mathrm{ng} / \mathrm{mL})$, alpha-fetoprotein $(0.7 \mathrm{ng} / \mathrm{mL})$, carbohydrate antigen $125(6.5 \mathrm{U} / \mathrm{mL})$, cancer antigen $15-3(0.5 \mathrm{U} / \mathrm{mL})$, carbohydrate antigen $19-9(4.7 \mathrm{U} / \mathrm{mL})$, and squamous cell carcinoma antigen $(0.8 \mathrm{ng} / \mathrm{mL})$ were all within the normal range. Magnetic resonance imaging showed a spaceoccupying lesion arising from the cervix with a diameter of approximately $5.5 \mathrm{~cm}$. The lesion extended into the vagina and parametrium (Figure 1). The tumor was classified as clinical Stage IIIB. A cervical biopsy specimen of the tumor, obtained for confirmation of the tumor type, was prepared and analyzed by a pathologist using IHC analysis.

\section{Pathologic findings}

Microscopically, hematoxylin and eosin (HE) staining of sections showed that the tumor was composed of diffuse, small, round blue-staining cells. The cells were fairly uniform and contained round nuclei with fine chromatin. Nucleoli were small and inconspicuous (Figure 2B). Mitotic figures and Homer Wright rosettes were occasionally encountered (Figure 2A). IHC staining for CD99 (Figure 3A),

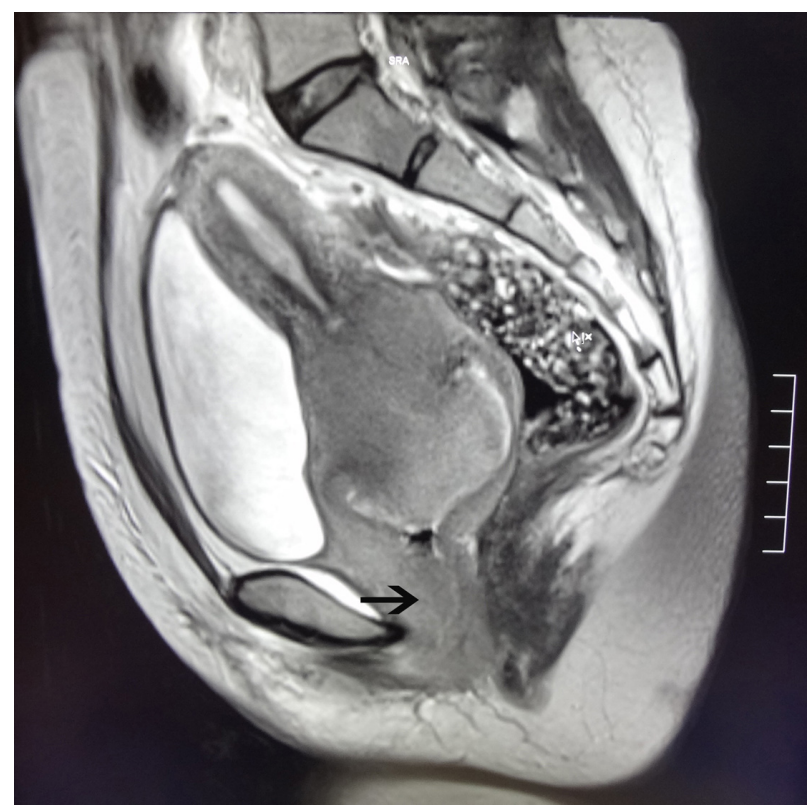

Figure I Magnetic resonance imaging scan showing a space-occupying lesion in the cervix that is invading the vagina (arrow). 


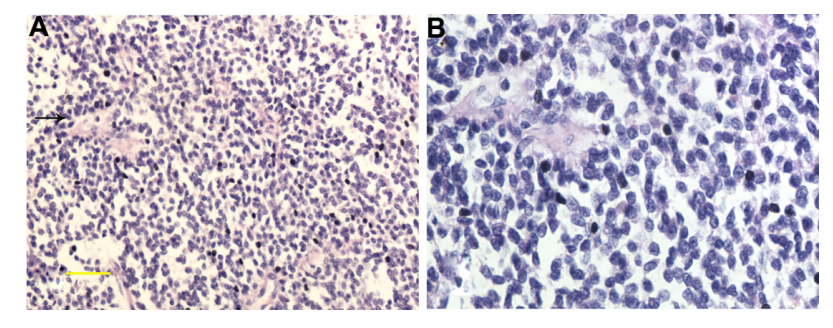

Figure 2 Histological findings. (A) Tumor composition - a diffused distribution of small round cells, with scattered Homer Wright rosettes (indicated by the arrow); (B) high-power microscopy revealed that the small round cells with round nuclei contained fine chromatin and there was limited clear or eosinophilic cytoplasm. Notes: $(A)$, magnification $\times 200$, scale bar, $50 \mu \mathrm{m}$; (B), magnification $\times 400$.

vimentin, CD117 (Figure 3B), NSE (Figure 3C), and CD56 (Figure 3D) was positive. Staining for all the other tested markers including pan-cytokeratin, TTF-1, TdT, CD45, CD38, desmin, myogenin, S-100, and HMB45 was negative. The Ki-67 labeling index was approximately $80 \%$.

\section{Discussion}

PNET is a relatively uncommon tumor that mainly occurs in the trunk, limbs, and retroperitoneum. ${ }^{4}$ Primary cervical PNETs are extremely rare and, to date and as far as we are aware, only nine cases have been reported in the literature. ${ }^{5-12}$ In contrast to patients with PNETs at other sites, which usually include children and young adults, the age of patients with primary cervical PNETs, as per these previous reports, ranged from 21 to 50 years (mean, 34 years). Of the nine patients, six who originally presented with localized disease were alive without evidence of recurrence or metastatic disease at an average follow-up time of 19 months (range, 5-50 months). Two patients died of disease, one 7 days

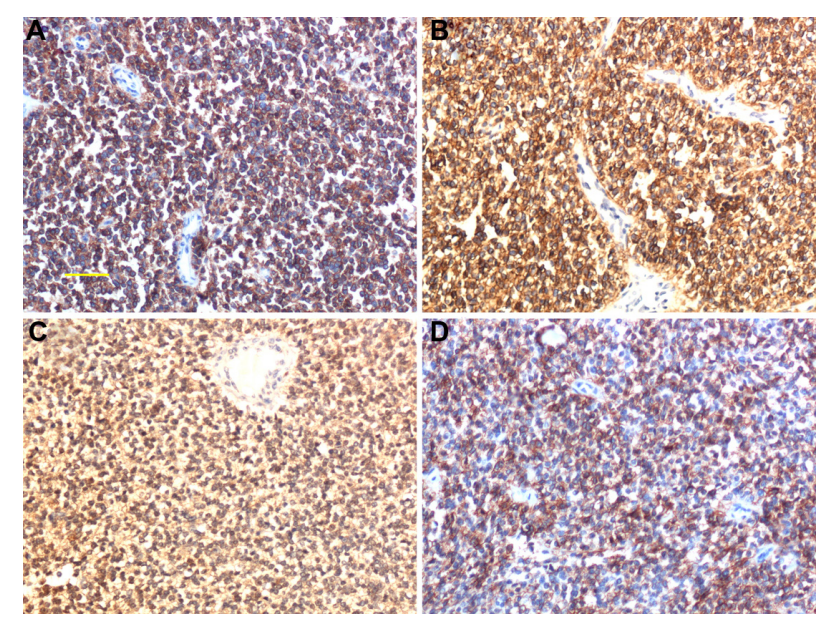

Figure 3 Immunohistochemical findings. (A) Strong reactivity in tumor cells with anti-cluster of differentiation (CD) 99. (B) CDI17; membranous and cytoplasmic staining. (C) Neuron-specific enolase, cytoplasmic staining $(\times 200)$. (D) CD56; membranous staining.

Notes: $($ A), magnification $\times 200$, scale bar $50 \mu \mathrm{m}$; (B) $-($ D), magnification $\times 200$. following surgery and the other 4.2 years after surgery. Survival details are not available for one patient. The patient presented in this paper was 27 years old, which is consistent with the literature.

PNET is mainly diagnosed based on histopathological morphology, IHC examination, and genetic testing. In the present case, conventional HE staining revealed that the tumor was composed of small round cells. Homer Wright rosettes, a characteristic manifestation of PNETs, are seen in approximately $30 \%$ to $70 \%$ of cases. ${ }^{13}$ While the presence of such rosettes can be used as an important basis to diagnose the tumor, IHC analysis is the primary means to diagnose PNETs. CD99 and vimentin staining is very characteristic and has a high sensitivity for PNET diagnosis. Occasionally, epithelial markers and desmin might show focal positivity, whereas the expression of neural markers such as NSE, S-100, neurofilaments, glial fibrillary acid protein, and CgA is uncertain. A characteristic chromosomal translocation $\mathrm{t}(11 ; 22)(\mathrm{q} 24 ; \mathrm{q} 12)$, resulting in EWS-FLI1 fusion, is observed in $85 \%$ of PNET cases; $;^{14,15}$ most of the remaining cases $(10 \%-15 \%)$ show fusion of the $E W S$ gene to a second member of the ETS family of genes, namely ERG. EWS-ERG fusion occurs as a result of the chromosomal translocation $\mathrm{t}(21 ; 22)(\mathrm{q} 22 ; \mathrm{q} 12) .{ }^{12}$

PNETs share characteristics with other small round blue-cell cancers including intra-abdominal desmoplastic small round cell tumor (IDSRCT), rhabdomyosarcoma, lymphoblastic lymphoma, endometrial stromal sarcoma or undifferentiated sarcoma, and small cell carcinoma. ${ }^{16}$ Membranous CD99 staining and focally positive NSE staining may be noted for PNETs. However, CD99 is not specific for PNETs; it can also be expressed in lymphoblastic lymphoma. ${ }^{17}$ In this case, based on negative staining for CD45 and TdT together with no evidence of lymphoepithelial lesions on histopathology, lymphoid system disease was excluded. IDSRCT is characterized by a nested pattern with prominent desmoplastic stroma. The tumor nests often show peripheral palisading with central necrosis. ${ }^{16}$ IDSRCT is diffusely positive for cytokeratin, desmin, and NSE. Rhabdomyosarcoma, in contrast to PNET, is strongly positive for desmin, myogenin, and myoD1. Endometrial stromal sarcoma and undifferentiated sarcoma are mainly composed of short spindle cells. Thick-wall spiral arteries are visible in the interstitial. In the present case, IHC analysis showed CD10 negativity and CD99 positivity, thereby excluding the diagnosis of stromal sarcoma. Negative staining for pan-cytokeratin and TTF-1 and positive staining for vimentin and CD117 also ruled out the possibility of small cell carcinoma. ${ }^{18,19}$ 
The most effective treatment for a PNET is surgery combined with chemotherapy and partial or systemic highdose radiotherapy. ${ }^{20}$ In the case of tumors without metastasis, surgical resection combined with chemotherapy is the treatment of choice. In the literature, five patients previously received only chemotherapy, one received radiotherapy alone, and the other three received a combination of chemotherapy and radiotherapy postoperatively. For chemotherapy regimens, Sato et $\mathrm{al}^{5}$ used cisplatin, cyclophosphamide, etoposide, and vincristine for six cycles. Horn et $\mathrm{al}^{6}$ used cisplatin and fluorouracil for 3 years after the initial therapy (for pulmonary metastasis). Snijders-Keilholz et al ${ }^{10}$ used six cycles of doxorubicin, ifosfamide, mesna, and etoposide preoperatively and vincristine, ifosfamide, and actinomycin for five cycles. Tsao et $\mathrm{al}^{13}$ used cyclophosphamide, doxorubicin (Adriamycin ${ }^{\circledR}$ Pfizer, New York, NY, USA), and vincristine and ifosfamide and etoposide for two cycles before surgery and two cycles after surgery.

In the present case, the clinical stage of the tumor was IIIB. The tumor had invaded the vaginal wall and was therefore too large for surgical resection. We used comprehensive treatment including partial radiotherapy (radiation dose, 45-55 Gy) combined with chemotherapy. We alternated between cyclophosphamide, Adriamycin, and vincristine with ifosfamide and etoposide chemotherapy every 3 weeks. After undergoing radiotherapy and chemotherapy, the patient experienced significant relief. At time of writing, 6 months after treatment, the patient is alive.

\section{Conclusion}

PNETs rarely occur as primary cervical neoplasms and have a poor prognosis. They can be diagnosed based on histopathological morphology, IHC examination, and genetic testing. Combined therapy including surgery, chemotherapy, and radiotherapy is an often-used treatment strategy. Here, we have described a case of PNET involving the cervix uteri in a 27 -year-old woman. The patient was treated with chemotherapy and radiotherapy and remains alive without disease after 6 months. However, further follow-up is needed to evaluate the prognosis of the patient.

\section{Disclosure}

The authors declare no conflicts of interest in this work.

\section{References}

1. Bartholow T, Parwani A. Renal primitive neuroectodermal tumors. Arch Pathol Lab Med. 2012;136(6):686-690.

2. von Bueren AO. CNS PNET molecular subgroups with distinct clinical features. Lancet Oncol. 2012;13(8):753-754.

3. Andrei M, Cramer SF, Kramer ZB, Zeidan A, Faltas B. Adult primary pulmonary primitive neuroectodermal tumor: molecular features and translational opportunities. Cancer Biol Ther. 2013;14(2):75-80.

4. Shimada H, Newton WA Jr, Soule EH, Qualman SJ, Aoyama C, Maurer HM. Pathologic features of extraosseous Ewing's sarcoma: a report from the Intergroup Rhabdomyosarcoma Study. Hum Pathol. 1988;19(4):442-453.

5. Sato S, Yajima A, Kimura N, Namiki T, Furuhashi N, Sakuma H. Peripheral neuroepithelioma (peripheral primitive neuroectodermal tumor) of the uterine cervix. Tohoku J Exp Med. 1996;180(2):187-195.

6. Horn LC, Fischer U, Bilek K. Primitive neuroectodermal tumor of the cervix uteri. A case report. Gen Diagn Pathol. 1997;142(3-4):227-230.

7. Cenacchi G, Pasquinelli G, Montanaro L, et al. Primary endocervical extraosseous Ewing's sarcoma/PNET. Int J Gynecol Pathol. 1998;17(1): $83-88$.

8. Pauwels P, Ambros P, Hattinger C, et al. Peripheral primitive neuroectodermal tumour of the cervix. Virchows Arch. 2000;436(1):68-73.

9. Malpica A, Moran CA. Primitive neuroectodermal tumor of the cervix: a clinicopathologic and immunohistochemical study of two cases. Ann Diagn Pathol. 2002;6(5):281-287.

10. Snijders-Keilholz A, Ewing P, Seynaeve C, Burger CW. Primitive neuroectodermal tumor of the cervix uteri: a case report - changing concepts in therapy. Gynecol Oncol. 2005;98(3):516-519.

11. Masoura S, Kourtis A, Kalogiannidis I, et al. Primary primitive neuroectodermal tumor of the cervix confirmed with molecular analysis in a 23-year-old woman: A case report. Pathol Res Pract. 2012;208(4): 245-249.

12. Farzaneh F, Rezvani H, Boroujeni PT, Rahimi F. Primitive neuroectodermal tumor of the cervix: a case report. J Med Case Rep. 2011;5:489.

13. Tsao AS, Roth LM, Sandler A, Hurteau JA. Cervical primitive neuroectodermal tumor. Gynecol Oncol. 2001;83(1):138-142.

14. Dedeurwaerdere F, Giannini C, Sciot R, et al. Primary peripheral PNET/ Ewing's sarcoma of the dura: a clinicopathologic entity distinct from central PNET. Mod Pathol. 2002;15(6):673-678.

15. Teicher BA, Bagley RG, Rouleau C, Kruger A, Ren Y, Kurtzberg L. Characteristics of human Ewing/PNET sarcoma models. Ann Saudi Med. 2011;31(2):174-182.

16. Hayes-Jordan A, Anderson PM. The diagnosis and management of desmoplastic small round cell tumor: a review. Curr Opin Oncol. 2011; 23(4):385-389.

17. Ozdemirli M, Fanburg-Smith JC, Hartmann DP, et al. Precursor B-Lymphoblastic lymphoma presenting as a solitary bone tumor and mimicking Ewing's sarcoma: a report of four cases and review of the literature. Am J Surg Pathol. 1998;22(7):795-804.

18. Pisick E, Skarin AT, Salgia R. Recent advances in the molecular biology, diagnosis and novel therapies for various small blue cell tumors. Anticancer Res. 2003;23(4):3379-3396.

19. Tsokos M, Alaggio RD, Dehner LP, Dickman PS. Ewing sarcoma/ peripheral primitive neuroectodermal tumor and related tumors. Pediatr Dev Pathol. 2012;15(Suppl 1):108-126.

20. Saif MW, Ng J, Chang B, Russo S. Is there a role of radiotherapy in the management of pancreatic neuroendocrine tumors (PNET)? JOP. 2012;13(2):174-176. 
OncoTargets and Therapy

\section{Publish your work in this journal}

OncoTargets and Therapy is an international, peer-reviewed, open access journal focusing on the pathological basis of all cancers, potential targets for therapy and treatment protocols employed to improve the management of cancer patients. The journal also focuses on the impact of management programs and new therapeutic agents and protocols on

patient perspectives such as quality of life, adherence and satisfaction. The manuscript management system is completely online and includes a very quick and fair peer-review system, which is all easy to use. Visit http://www.dovepress.com/testimonials.php to read real quotes from published authors.

Submit your manuscript here: http://www.dovepress.com/oncotargets-and-therapy-journal 